\section{Is it Possible to Create a Uni- versal Cure for Pneumonia?}

\section{Igor Klepikov*}

Department of Pediatric Surgery, Tel Aviv University, Renton, Washington, USA

Antibiotics alone remain today the guiding principle of the treatment of many inflammatory diseases including Acute Pneumonia (AP). If this principle of treatment has produced the expected results, the discussion of this topic would not be required. Therefore, the published results show not only the negative dynamics of development of purulent complications of the AP, but also the lack of promising strategies to address this problem [1-4]. Pediatric pleural empyema has increased substantially over the past 20 years and reasons for this rise remain not fully explained [5]. In developed (!) countries from $9.5 \%$ to $42 \%$ of patients with pneumonia are received in the hospital due to the ineffectiveness of primary treatment [6]. However, the transfer of patients to hospital does not improve the results of treatment. Parapneumonic effusions or empyema affect $2 \%$ to $12 \%$ of children with pneumonia, and up to $28 \%$ of those requiring hospitalization [7].

Unfortunately, the modern scientific explanation and recommendations for treatment of AP and prevention of its complications direct practical medicine only to suppress microflora. Therefore, the main, and in many patients, the only means of treatment are antibiotics. Such recommendations for certain time periods based on the allegations of a predominant participation of specific bacteria in the development of AP. In recent years, this bogey was Streptococcus pneumonia. However, the well-known facts and results do not confirm the specificity of the etiology of AP. Most of the patients with AP heal without any verification of the etiology of the disease. Therefore, discussions on the etiology of inflammation in these patients in the absence of concrete results are simply groundless. Bacteriological examination of material from the zone of inflammation becomes available in case of purulent complications. Only the results of these studies indicate the participation of the most diverse microflora. Scientific medicine has received the most serious blow to its reputation after years of

*Corresponding author: Igor Klepikov, Department of Pediatric Surgery, Tel Aviv University, Renton, Washington, USA, Tel: +1 2069209643; E-mail: iklepikov@yahoo.com

Citation: Klepikov I (2017) Is it Possible to Create a Universal Cure for Pneumonia? J Pulm Med Respir Res 3: 013.

Received: September 25, 2017; Accepted: October 24, 2017; Published: November 24, 2017 total pneumococcal vaccination of the population in developed countries. So, a statistically significant increase in suppurative complications AP, contrary to the prediction of decline, is still without a proper scientific explanation. Among children $\leq 18$ years of age, the annual empyema-associated hospitalization rates increased almost $70 \%$ between 1997 and 2006, despite decreases in the bacterial pneumonia and invasive pneumococcal disease rates. Pneumococcal conjugate vaccine is not decreasing the incidence of empyema [8].

Despite decrease in pneumococcal diseases among Utah children, complicated pneumonia/empyema has increased during the 7-valent pneumococcal conjugate vaccine era. The causes on increasing rates of empyema are unclear [9].

The discovery of antibiotics was outstanding achievement in the past century. Their practical applications have saved the lives of a huge number of patients. Therefore, the importance of antibiotics in complex medical care to various categories of patients is not in doubt. At the same time, over several decades the use of antibiotics in clinical practice there is such a major transformation that requires a dramatic revision of the strategy in many specific areas and revaluation of the role and place of these drugs in the set of medical care. The first stunning results of antibiotic use were so impressive that deservedly gave rise to the opinion of their exclusivity and elevated them to the rank of a panacea for many diseases. But time passed and microflora, as a biological entity, able to adapt to this "medical aggression". Antibiotic-resistant microbe strains began to appear as a natural consequence of such exposure (because the absolute sterilization of living biological world is impossible, isn't it?). Today we already have quite a long list of such resistant strains and the list is constantly updated (Polymicrobial cap). The history of all these transformations has a second side: a decline in the effectiveness of antibiotics and the rise of microbial resistance to them has been a powerful incentive for pharmacists to develop new and more active and powerful drugs. Therefore, at present it is impossible to enumerate from memory all known antibiotics and the number of generations. And here is exposed the dilemma, which cannot be invisible, and which requires evaluation and decision. On the one hand, medical practice regularly receives new and more powerful antibiotics. On the other hand, updating the arsenal of antimicrobial agents does not prevent the growth of purulent complications of AP.

Currently, the image of a "new" infectious threat arises. Respiratory viruses, rather than bacterial pathogens, were most commonly detected in children hospitalized with pneumonia. This ground-breaking study shows how badly we need faster, less-expensive diagnostic tests for doctors to accurately diagnose the cause of pneumonia so they can effectively treat it [10]. "The results help define the role of viruses as major players in pediatric pneumonia and shows a need for new therapies that can reduce the severity of viral pneumonia", says Chris Stockmann, co-investigator and senior research analyst at the University of Utah. Among children diagnosed with pneumonia, viral infections were much more common than bacterial infections (73 vs. 15\%), and Respiratory Syncytial Virus (RSV) was the most commonly detected pathogen [11]. It is significant that the frequency of detection of these viruses in healthy people is not statistically different from their etiological role in acute pneumonia. 
If you stick to the old approach in the treatment of AP, the new information about the etiology of the disease puts the pharmacists a new challenge: the need to develop and create unique group of antiviral drugs. It should be noted that the principles of solving the problem remain the same and changes can apply only to tactical efforts. Beginning this way will be a new round of competitive race between pathogens and pharmaceuticals. Modern treatment results in AP show that the previous round of this competition ends not in favor of the creators of the drugs. So, before you start intensive work in this direction, we should recall well-known facts about the nature of the AP.

It is well known that pneumonia is not a contagious of infectious diseases and not in need of appropriate measures for the health and safety [12]. It is also common knowledge that many people are carriers of the entire spectrum of microbes that are pathogens of AP. These people feel great and do not need medical attention. In other words, the presence of a microbe is insufficient for the occurrence of inflammation in the lung. Such a conclusion is not a revelation to many experts. Please note, we are not talking about such dangerous infections as Plague, Cholera, Typhus, Smallpox, etc., can cause a terrible epidemic. Today the problem lies elsewhere. The current generation of physicians are educated in the fear of the banal microflora and worship before antibiotics and acts in accordance with their education and existing requirements.

Doctors in training at the University are aware of many medical axioms,which have a scientific basis and proof, but this knowledge does not affect their general idea about the nature of the AP. As an example, the following are some of such axioms are of direct relevance to the development and dynamics AP.

1. The body's response to any stimulus, including the initiation of inflammation, is highly individual and unique.

2. The basis for the inflammatory transformation of the body tissue is a vascular reaction with a specific stage sequence.

3. Small and big circles of blood circulation not only have a direct relationship, but an inverse relationship.

4. Among the nonspecific forms of inflammation, AP is the only process occurring in the system of lesser circulation.

5. The same medical procedure can have different effects on inflammation in the small or big circles of blood circulation [13$16]$.

Therefore, it follows from all the foregoing, the problem of guaranteed cure of AP and the prevention of its purulent complications should be carried out through a revision of the understanding of the mechanisms of emergence and subsequent development of the disease. However, from my point of view. There is another dilemma. On the one hand, the new doctrine AP not only created and argued for more original research, but also clinically tested with excellent results. A detailed description of the results of this work can be found in a published book- Igor Klepikov - "Acute pneumonia: a new look at the old problem ", Lambert Academic Publishing, 2017, ISBN (9783-330-35250-6).

On the other hand, the most difficult task in the field of care for patients with AP will change existing stereotypes. The impact of the prevailing concept of AP on the mindset of a vast number of experts will be impossible to fix in a short time. This paradigm remained dominant for several decades, accompanying health professionals with the University throughout their career. The obvious argument for the introduction of new educational programs under section AP is the modern statistics of outcomes in these patients and complete stagnation in the explanation of failures and negative trends in these results. How much time may require such retraining, it is difficult to predict. One thing is quite clear, the beginning of this work cannot be postponed.

\section{References}

1. Klepikov I (2017) Acute pneumonia in children: The price of illusions and delusions. J Pediatr Care 3: 1-2.

2. Klepikov I (2017) Why need a new concept of acute pneumonia. J Tradit Med Clin Natur 6: 212.

3. https://gehealthcloud.devpost.com/forum topics/6433-tablet-against-pneumonia-modern-delusion-igor-klepikov-md-professor

4. Klepikov I (2017) The new doctrine of acute pneumonia-the key to solve the problem. EC Psychology and Psychiatry 4: 138-139.

5. Elemraid MA, Thomas MF, Blain AP, Rushton SP, Spencer DA, et al. (2015) Risk factors for the development of pleural empyema in children. Pediatr Pulmonol 50: 721-726.

6. Pabary R, Balfour-Lynn LM, (2013) Complicated pneumonia in children. Breathe 9: 210-222.

7. Janahi IA, Fakhoury K (2017) Epidemiology; clinical presentation; and evaluation of parapneumonic effusion and empyema in children. Wolters Kluwer, Alphen aan den Rijn, Netherlands.

8. Wikkelsø AJ, Edwards HM, Afshari A, Stensballe J, Langhoff-Roos J, et al. (2015) Pre-emptive treatment with fibrinogen concentrate for postpartum haemorrhage: randomized controlled trial. Br J Anaesth 114: 623-633.

9. Ampofo K, Pavia AT, Stockmann CR, Blaschke AJ, Weng HY, et al. (2011) Evolution of the epidemiology of Pneumococcal Disease among Utah Children through the vaccine era. Pediatr Infect Dis J 30: 11001103.

10. http://www.cdc.gov/media/releases/2015/p0225-pneumonia-hospitalizations.html

11. https://healthcare.utah.edu/publicaffairs/news/2015/02/022515_Childhood_Pneumonia.php

12. Klepikov I (2017) Acute pneumonia in children - Illness or infection? J Infect Non Infect Dis 3: 1-2.

13. Klepikov I (2017) The effect of intravenous infusion on the dynamics of acute pneumonia. EC Pulmonology and Respiratory Medicine 4: 15-20.

14. Klepikov I (2017) The meaning of pulmonary reflexes in the pathogenesis of acute pneumonia. Intern Med 7: 232.

15. Klepikov I (2017) The role of cardiovascular disorders in the pathogenesis of acute pneumonia. Journal of Cardiology \& Cardiovascular Therapy $4: 1-2$.

16. Klepikov I (2017) Cupping therapy in the 21st century? - Why not!? Journal of General and Emergency Medicine 2: 1-3. 